Adequate ULT was defined as a mean daily dose of at least $300 \mathrm{mg}$ of allopurinol and a duration of therapy of at least 2 years. Patients that had received adequate ULT were matched using propensity score on the basis of baseline eGFR and length of follow-up time to patients that received non-adequate ULT. Change from baseline in SU and eGFR was calculated and compared between groups.

Results: We identified 5433 patients with an incident gout diagnosis during 2000-2012 (and no gout diagnosis or prescription for ULT during 1997-1999).

Of these, $2393(44 \%)$ received at least one prescription for allopurinol. SU was measured at some time point after initiation of ULT in $58 \%$ of patients. Adequate ULT as defined above was prescribed for 154 patients $(3 \%)$, of these, $112(73 \%)$ had a SU measurement at some time point after initiation of therapy and $35(23 \%)$ had such a measurement done within 6 weeks of starting treatment.

Matched controls could be identified for 109 of the patients with adequate ULT. Mean urate and eGFR at the start of therapy and end of follow up for the group with adequate ULT treatment and the controls are shown in table 1.

Table 1. Values are mean (SD) or $n(\%)$

Age

Female, $\mathrm{n}(\%)$

Allopurinol daily dose in $\mathrm{mg}$

Baseline s-urate, $\mu \mathrm{mol} / \mathrm{L}$

Baseline eGFR, $\mathrm{ml} / \mathrm{min}$

Length of follow-up, years

$\Delta \mathrm{s}$-urate, (baseline compared to last

available value)

$\triangle$ e-GFR (baseline compared to last

available value)

Last eGFR worse than baseline

measurement, $\mathrm{n}(\%)$

$\begin{array}{ccc}\begin{array}{c}\text { Adequate ULT, } \\ \mathbf{n}=109\end{array} & \begin{array}{c}\text { Matched controls, } \\ \mathbf{n}=109\end{array} & \mathbf{p} \text {-value } \\ 66(14) & 68(13) & 0.19 \\ 25(23) & 38(35) & 0.05 \\ 359(61) & 119(63) & <0.0001 \\ 511(111) & 509(100) & 0.91 \\ 66(21) & 66(21) & 0.84 \\ 4.2(3.5) & 5.2(3.9) & 0.05 \\ -168(153) & -78(125) & <0.0001 \\ -1(15) & -5(16) & 0.039 \\ & & \\ 50(46) & 67(61) & 0.021 \\ & & \\ \end{array}$

Conclusion: ULT was prescribed to less than half of the patients identified. Adequate ULT was rare in clinical practice during the time period studied. Urate monitoring occurred in less than half of ULT-treated patients. The patients with adequate ULT achieved greater lowering of serum urate than matched controls and were more likely to maintain unchanged renal function over time.

Acknowledgement: Göran Rörvall, database consultant.

Disclosure of Interests: Valgerdur Sigurdardottir: None declared, Lennart T.H. Jacobsson Consultant for: LJ has received lecture and consulting fees from Pfizer, Abbvie, Novartis, Eli-Lily and Janssen, Anna Svärd: None declared, Mats Dehlin: None declared

DOI: 10.1136/annrheumdis-2019-eular.6255

\section{SAT0446 GOUT IN THE US: SIGNIFICANT ASSOCIATIONS WITH CARDIOVASCULAR AND RENAL DISEASE HOSPITALIZATIONS}

Gurkirpal Singh ${ }^{1,2}$, Maanek Sehgal ${ }^{3}$, Alka Mithal ${ }^{4} .{ }^{1}$ ICORE, Woodside, United States of America; ${ }^{2}$ Stanford University, Palo Alto, United States of America; ${ }^{3}$ UCLA, Los Angeles, United States of America; ${ }^{1}$ ICORE, Woodside, United States of America

Background: Gout is a disorder of uric acid metabolism and often presents as acute severe joint pain. Previous work from our group suggests that all-cause hospitalizations in patients with gout in the United States (US) have significantly increased by $410 \%$ in the last 22 years, almost hundred-fold of the $4.8 \%$ increase in US population all-cause hospitalization rate in the same time-period. The current study focused on identifying potential reasons for the excess hospitalizations.

Objectives: To compare the primary reasons for hospitalizations in patients with gout to those in age-matched patients without gout in the US in 2016.

Methods: The Nationwide Inpatient Sample (NIS) is a stratified random sample of all US community hospitals. It is the only US national hospital database with information on all patients, regardless of payer, including persons covered by Medicare, Medicaid, private insurance, and the uninsured. We examined all inpatient hospitalizations in the NIS in 2016 among patients 40 years or older with a primary or secondary diagnosis of gout and compared them to total all-cause hospitalizations in patients without a gout diagnosis in the same age group during the same period. Over 69,800 ICD 10 diagnoses codes were collapsed into a smaller number of clinically meaningful categories, consistent with the Centers for Disease Control (CDC) Clinical Classification Software. The top 15 primary causes for hospitalization were evaluated and compared between the two cohorts.

Results: In 2016, there were 892,379 all-cause hospitalizations in the US in patients with gout with $14,135(1.6 \%)$ of these for a primary diagnosis of gout. The primary diagnoses for 878,244 hospitalizations in patients with gout were compared with those in 21.9 million hospitalizations in the general US population without gout. The most common primary cause of hospitalizations in both cohorts was septicemia $(7.8 \%$ in gout vs. $7.4 \%$ in general population). Significant differences were seen in several other categories. For example, acute renal failure was twice as frequent among gout patients $(39,455$ hospitalizations, $4.5 \%)$ compared to the general population (458,920 hospitalizations, 2.1\%) (Relative Risk (RR) $2.11,95 \%$ Cl 2.08 - 2.13, p<0.0001). Hospitalizations for "hypertensive complications and secondary hypertension" were also higher $(35,435,4.0 \%$ in gout vs $370,400,1.7 \%$ in general population, $\mathrm{RR} 2.35,95 \% \mathrm{Cl} 2.32-2.37$ $\mathrm{p}<0.0001)$. Hospitalizations from gastrointestinal bleeding were $30 \%$ more common in gout patients, perhaps associated with concomitant NSAID treatments (Figure).

Conclusion: Among patients with gout, a far greater proportion of serious hospitalizations are related to renal and cardiovascular complications as compared to age-matched general population. This calls for an increased awareness and management of serious co-morbid conditions in patients with gout.

\section{REFERENCES}

[1] Singh G, Mithal A, Mithal A. NOT JUST A SWOLLEN BIG TOE: INCREASING ALL-CAUSE HOSPITALIZATIONS IN PATIENTS WITH GOUT IN THE UNITED STATES: 1993-2014: Presented at EULAR Congress 2017

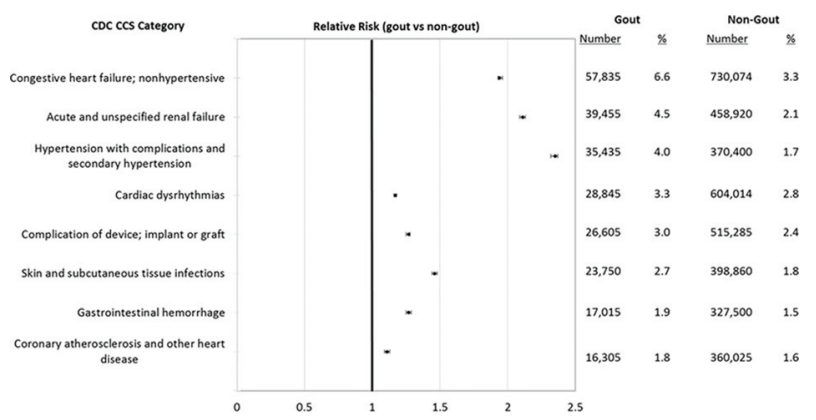

Figure

Primary reasons for comorbid hospitalizations more common in patients with gout compared with age-matched patients without gout, classified by Centers for Disease Control Clinical Classification Software (CDC CCS). Acknowledgement: Supported in part by an unrestricted grant from Horizon Pharma

Disclosure of Interests: Gurkirpal Singh Shareholder of: Merck, Pfizer Grant/research support from: Horizon, Acorda, UCB, Consultant for: Horizon, Acorda, Sancilio, Unipharm, Maanek Sehgal: None declared, Alka Mithal: None declared

DOI: 10.1136/annrheumdis-2019-eular.2578

\section{SAT0447 MANAGEMENT OF GOUT: AN AUDIT IN PRIMARY CARE GENERAL PRACTICE IN LEICESTERSHIRE, UNITED KINGDOM}

Ahmed Aboo ${ }^{1}$, Larry Goodyer ${ }^{2}$, Neena Lakhani ${ }^{1}$, Muhammed Hadi ${ }^{3}$, Kehinde Sunmboye ${ }^{4} .1$ DeMonfort University, Leicester School of Pharmacy, Leicester, United Kingdom; ${ }^{2}$ DeMonfort University, Leicester school of Pharmacy, Leicester, United Kingdom; ${ }^{3}$ University of Birmingham, Institute of Clinical sciences, Birmingham, United Kingdom; ${ }^{4}$ University Hospitals Of Leicester, Department of Rheumatology, Leicester, United Kingdom

Background: Both acute and long-term management of gout in primary care in UK is reportedly suboptimal ${ }^{1}$, with patients rarely being offered lifestyle advice or urate Lowering Therapy (ULT). Further, ULT is often not titrated according to the patient's serum uric acid (sUA) levels, so that they fail to achieve target sUA levels.

Objectives: To audit and evaluate the management of gout in patients in a primary care setting against the current guidelines treat to target guidelines set by the British Society of Rheumatology (BSR)2. 
Methods: Audit criteria were derived from the latest BSR gout guideline (Hui et al; 2017). A randomised sample of adult patients with a read code for the diagnosis of gout from Jan 2006-Jan 2018 was chosen from six large general practices in Leicestershire County of the United Kingdom. The data collected included demographics, provision of patient information, management of acute attacks and prophylactic treatment, screening of appropriate co-morbidities, dosing of urate-lowering therapy (ULT) and titration of doses against measurement of uric acid levels.

Results: Data was obtained for 861 patients The mean age was 60 years and $91 \%$ were male, $21.5 \%$ were recorded as being provided with written information about gout and $60.5 \%$ of patients were treated with NSAIDs and COXIBs for acute attacks of gout. When colchicine was prescribed to patients, $71 \%$ had no dose recorded in their clinical records. $323(37.5 \%)$ of patients were prescribed a ULT and the recorded starting dose of allopurinol was $100 \mathrm{mg}$ daily for $73.8 \%$. Titration of subsequent allopurinol doses was recorded in only $21 \%$ of patients. 539 patients $(62.6 \%)$ had no record of a serum urate level check after starting ULT. Conclusion: Clinical records indicate that the management of gout by UK General Practitioners in Primary Care is suboptimal in concordance with the BSR guidelines. It was clear that general practices did not employ the treat to target strategy. There is a clear need for increased GP awareness and adherence to the BSR guidelines in order to optimise deficient areas of care, particularly in patient education, initiation and titration of ULT and monitoring of serum urate levels in gout patients. Appropriate patient recording templates are needed so that key information is captured during a patient consultation in order to enable medicines optimisation for those with gout. Most aspects of gout management in primary care did not concord well with published BSR guidelines.

\section{REFERENCES}

[1] Kuo, C., Grainge, M.J., Mallen, C., Zhang, W. and Doherty, M. (2014) Rising burden of gout in the UK but continuing suboptimal management: a nationwide population study. Annals of the Rheumatic Diseases, [online] 74(4) pp.661-667.

[2] Hui, M., Carr, A., Cameron, S., Davenport, G., Doherty, M., Forrester, H., Jenkins, W., Jordan, K., Mallen, C., Mcdonald, T., Nuki, G., Pywell, A., Zhang, W. and Roddy, E. (2017) The British Society for Rheumatology Guideline for the Management of Gout. Rheumatology, [online] 56 (7) pp. e1-e20.

Disclosure of Interests: None declared

DOI: 10.1136/annrheumdis-2019-eular.8137

\section{SAT0448 ATP IS THE SECOND KEY SIGNAL OF GOUT FLARE BESIDES MSU}

Hong-Liang Zhang ${ }^{1}$, Zi-Wen Zhu ${ }^{2}$, Jinhui Tao ${ }^{1,3}$, Yu-Jie Tang ${ }^{1}$, LI Xin-Ya ${ }^{1}$, LI Lin ${ }^{2}$, Qun-Qun Lu'. ${ }^{1}$ Division of Life Sciences and Medicine, University of Science and Technology of China, hefei, China; ${ }^{2}$ Anhui Medical University Affiliated Provincial Hospital, hefei, China; ${ }^{3}$ Anhui Medical University Affiliated Provincial Hospital, hefei, China

Background: Gout is an inflammatory disease associated with hyperuricemia and characterized by recurrent arthritis. In previous study, MSU which generated by hyperuricemia was recognized by the toll-like receptor and NOD receptor of the intrinsic immune system, then activated the NALP3 inflammasome to induce the secretion of IL-1beta, causing gout. However, this mechanism cannot explain why most patients with hyperuricemia do not have gout attacks in clinical practice, suggesting that there may be other pathogenic signals in the flare of gout. Our team previously found that P2X7R might play a key regulatory role in the pathogenesis of gout ${ }^{[1]}$. What's more, single nucleotide polymorphisms associated with P2X7R function regulate the onset of gouty arthritis ${ }^{[2]}$, suggesting that ATP, as the ligand of P2X7R, may be a second key signal in addition to MSU to stimulate gout attack.

Objectives: To understand whether ATP act as a key factor in gout development besides MSU.

Methods: The rat hyperuricemia model was prepared by intraperitoneal injection of the uratase inhibitor oxazinic acid. Followed by ATP injection into the tail vein to observe the incidence of arthritis and compared with the Coderre's method-established gouty arthritis ${ }^{[3]}$.

Results: Forty hyperuricemia rats were prepared and $0.5 \mathrm{ml}$ ATP (10mM) solution was injected into the tail vein of the rat respectively, about $95 \%$ (38 rats) developed spontaneous arthritis. This type of spontaneous arthritis is very different from the Coderre's method in the following aspects: (1) The onset time of spontaneous arthritis is faster. The time of joint swelling in spontaneous arthritis peaked at $6.0 \pm 2.0$ hours after ATP injection, while it took 2 to 3 days for arthritis caused by local injection of
MSU to reach its peak. (2) The affected joints were different: The Coderre's method joints swelling only at the MSU injection site. Spontaneous arthritis can involve multiple joints, in which $71 \%$ have one joint affected, $21 \%$ have two joints affected and $8 \%$ with three or more than three joints affected by redness and swelling. (3) Distinct histopathologic characteristic: In Coderre's method, lymphocytes caused by local injection account for about $60 \%$, with fewer neutrophils; In spontaneous arthritis neutrophils account for more than $90 \%$ and lymphocytes are less. (4) Different sources of MSU: there is local MSU in spontaneous arthritis, which is associated with hyperuricemia; the local presence of MSU in Coderre's arthritis was artificially injected.

Conclusion: The spontaneous arthritis due to the synergistic effect of ATP and MSU is consistent with the characteristics of human gout arthritis, suggesting that ATP is the key second signal besides MSU to stimulate gout attack.

\section{REFERENCES}

[1] Tao JH, Zhang Y, Li XP. P2X7R: A potential key regulator of acute gouty arthritis. Semin Arthritis Rheum. 2013; 43(3): 376-380.

[2] Tao JH, Cheng M, Tang JP, et al. Single nucleotide polymorphisms associated with P2X7R function regulate the onset of gouty arthritis. PLoS One. 2017 Aug 10;12(8):e0181685

[3] CoderreTJ, Wall PD.Ankle joint urate arthritis (AJUA) in rats: An alternative animal model of arthritis to that produced by Freund's adjuvant. Pain. 1987; 28(3):379-93.

Acknowledgement: This work was supported by grants from the National Natural Science Foundation of China (81771774) and the Anhui Provincial Natural Science Foundation (1708085MH191)

Disclosure of Interests: None declared

DOI: 10.1136/annrheumdis-2019-eular.7510

\section{SAT0449 EFFECT OF CHOLESTEROL AND TRIGLYCERIDE ON THE FREQUENCY OF GOUT ATTACKS}

LI Lin ${ }^{1}$, Qun-Qun Lu ${ }^{1}$, Jinhui Tao ${ }^{2}$, Hong-Liang Zhang ${ }^{2}$, Yu-Jie Tang ${ }^{2}$, LI Xin-Ya ${ }^{2}$ Zi-Wen Zhu' ${ }^{1}{ }^{1}$ Anhui Medical University Affiliated Provincial Hospital, Department of Rheumatology and Immunology, hefei, China; ${ }^{2}$ The First Affiliated Hospital of USTC, Division of Life Sciences and Medicine, University of Science and Technology of China, Department of Rheumatology and Immunology, hefei, China

Background: Gout is an autoinflammatory disease characterized by hyperuricemia and recurrent arthritis. ATP and MSU synergistically activate NALP3 inflammasome to induce the secretion of IL-1B, leading to the onset of gouty arthritis, and P2X7R plays a key role in gout ${ }^{[1,2]}$. This mechanism above can explain the clinical phenomenon that some patients with hyperuricemia never suffer gouty arthritis, however, it cannot explain why the frequency of gout attacks increases as the course prolongs. Cholesterol or triglyceride can activate the innate immune and induce inflammatory response. It is speculated that Cholesterol and triglyceride levels can increase with the duration of gout, and they may reduce the threshold of gout attacks.

Objectives: To demonstrate the effect of elevated cholesterol and triglyceride on the onset of gout.

Methods: A cohort study was performed to observe the difference of arthritis episodes between the high cholesterol group and the normal, the high triglyceride group and the normal in gout patients. The frequency of gout attacks was compared using statistical methods of independent sample test and paired sample test between the two groups.

Results: A cohort study was performed to observe the difference of arthritis episodes between the high cholesterol group and the normal, the high triglyceride group and the normal in gout patients. The frequency of gout attacks was compared using statistical methods of independent sample test and paired sample test between the two groups.

RESULTS: A total of 68 patients with gout were observed. Among them, 21 were in normal cholesterol group, 13 in elevated group, 21 in normal triglyceride group and 13 in elevated group. The results of the independent sample test between the two groups are as follows: (1) The frequency of gout attacks between the normal cholesterol group and the elevated group is statistically significant within three months, six months and one year $(0.81 \pm 0.60$ vs $1.77 \pm 0.83, Z=-3.200, P=0.001 ; 1.14 \pm 0.73$ vs $3.15 \pm 2.15, \quad Z=-3.430, \quad P=0.001 ; \quad 1.43 \pm 0.81 \quad$ vs $4.77 \pm 3.44, \quad Z=-3.199$, $P=0.001)$. (2) The frequency of gout attacks between the normal triglyceride group and the elevated group is statistically significant within three months, six months and one year $(0.81 \pm 0.60$ vs $1.54 \pm 0.97, Z=-2.359$, $P=0.018 ; 1.14 \pm 0.73$ vs $2.38 \pm 1.66, Z=-2.417, P=0.016 ; 1.43 \pm 0.81$ vs 3.54 $\pm 2.50, Z=-3.005, P=0.003)$. The results of the paired sample test between 